\title{
Change in cognition and body mass index in relation to preclinical dementia
}

\author{
Ida K. Karlsson ${ }^{1,2}$ \\ Yiqiang Zhan ${ }^{3}$ \\ Margaret Gatz ${ }^{1,4}$ \\ Chandra A. Reynolds ${ }^{5}$ \\ Anna K. Dahl Aslan ${ }^{1,2,6}$
}

${ }^{1}$ Department of Medical Epidemiology and Biostatistics, Karolinska Institutet, Stockholm, Sweden

${ }^{2}$ Institute of Gerontology and Aging Research Network - Jönköping (ARN-J), School of Health and Welfare, Jönköping University, Jönköping, Sweden

${ }^{3}$ Institute of Environmental Medicine, Karolinska Institutet, Stockholm, Sweden

${ }^{4}$ Center for Economic and Social Research, University of Southern California, Los Angeles, California, USA

${ }^{5}$ Department of Psychology, University of California, Riverside, California, USA

${ }^{6}$ School of Health Sciences, University of Skövde, Skövde, Sweden

\section{Correspondence}

Ida K. Karlsson, Department of Medical Epidemiology and Biostatistics, Karolinska Institutet, SE-1177 Stockholm, Sweden.

Email: ida.karlsson@ki.se

Chandra A. Reynolds and Anna K. Dahl Aslan contributed equally to this study.

\begin{abstract}
Introduction: To study if declining cognition drives weight loss in preclinical dementia, we examined the longitudinal association between body mass index (BMI) and cognitive abilities in individuals who did or did not later develop dementia.

Methods: Using data from individuals spanning age 50 to 89, we applied dual change score models separately in individuals who remained cognitively intact $(n=1498)$ and those who were diagnosed with dementia within 5 years of last assessment $(n=459)$. Results: Among the cognitively intact, there was a bidirectional association: Stable BMI predicted stable cognition and vice versa. Among individuals who were subsequently diagnosed with dementia, the association was unidirectional: Higher BMI predicted declining cognition but cognition did not predict change in BMI.

Discussion: Although BMI and cognition stabilized each other when cognitive functioning was intact, this buffering effect was missing in the preclinical dementia phase. This finding indicates that weight loss in preclinical dementia is not driven by declining cognition.
\end{abstract}

\section{KEYWORDS}

body mass index, cognition, longitudinal, preclinical dementia, weight change

\section{BACKGROUND}

Pathophysiological changes in dementia start many years before clinical manifestations are seen. During this preclinical phase, cognitive abilities decline progressively, and are followed by a decline in functional abilities. ${ }^{1}$ Unintentional weight loss appears common during the preclinical dementia phase. $^{2}$ This weight loss is hypothesized to explain the "obesity paradox," where a high body mass index (BMI) in midlife has been robustly and positively associated with dementia, whereas a higher BMI in late-life may instead be associated with lower dementia risk. ${ }^{3-5}$ BMI generally increases from early adulthood through age 65 , after which it levels off to then start to decrease at around age $80 .^{6}$
These fluctuations appear to be more pronounced among individuals who are later diagnosed with dementia, where BMI tends to increase more at earlier ages, but then starts to fall more sharply $\approx 10$ years before diagnosis. $^{2}$ It is thus plausible that BMI can act both as a risk factor and a prodromal sign of dementia, depending on the timing of measurement and on longitudinal weight trajectories.

The same paradoxical pattern has been observed for BMI and cognition, where a higher midlife BMI is associated with lower cognitive ability in late life, but the association between late-life BMI and cognitive ability is less clear and may be in the inverse direction. ${ }^{4,5}$ It is important to note that the associations between late-life BMI and cognitive abilities may differ in normative cognitive aging and preclinical 
dementia, where it is not established whether declining cognitive abilities are a driver of weight loss. To investigate this question, we aimed to study the longitudinal and dynamic association between BMI and cognitive abilities, including the direction of effect, separately in individuals who later developed dementia and those who remained cognitively intact. We thereby hope to better understand the complex relationship between overweight, cognitive abilities, and dementia, and the nature of the obesity paradox.

\section{METHODS}

\subsection{Study population}

We used data from three longitudinal studies of aging within the Swedish Twin Registry, ${ }^{7}$ with rich longitudinal cognitive data, dementia diagnoses, and linkages to healthcare registers, making them ideal for the questions under study. The Swedish Adoption/Twin Study of Aging (SATSA) ${ }^{8}$ consists of 859 individuals from same-sex twin pairs, who participated in up to 10 in-person testing phases between 1986 and 2014. Aging in Women and Men (GENDER) ${ }^{9}$ includes 496 individuals from 248 opposite-sex twin pairs, who participated in up to three inperson testing phases conducted on a 4-year rolling schedule between 1995 and 2005. Origins of Variance in the Oldest Old: Octogenarian Twins (ОСTO-Twin) ${ }^{10}$ consists of 702 individuals from 351 same-sex twin pairs over age 80 , who participated in up to five in-person testing occasions conducted on a 2-year rolling schedule between 1991 and 2001. The in-person testing phases were conducted in a similar manner across the three studies, and included a health examination, cognitive assessments, and an extensive interview. We could thus pool individuals from the three studies, yielding a study sample of 2057 individuals.

All participants provided informed consent, and the studies were approved by the regional ethics board in Stockholm.

\subsection{BMI measurements}

Height and weight were measured by trained research nurses as part of the health examinations during each in-person testing occasion. The measures have been thoroughly examined for outliers, both quantitatively and visually, by plotting individual trajectories over time as described in detail previously. ${ }^{11}$ BMI was calculated as kilograms divided by height (in meters) squared. BMI measures below 15 or above 55 and unrealistic changes over a short time period were set to missing, but BMI was otherwise allowed to vary. Individual BMI trajectories across age showed comparable patterns in the three studies (Figure S1).

\subsection{Cognitive measures}

During each testing occasion, cognitive tests were performed covering four domains: verbal abilities (Synonyms), spatial abilities (Block

\section{RESEARCH IN CONTEXT}

1. Systematic review: The authors searched online databases (eg, PubMed) for work focused on changes in body weight and cognitive ability in the preclinical dementia phase. Although it is postulated that the inverse association between a higher body mass index (BMI) in late life and dementia risk stems from unintentional weight loss in the preclinical dementia phase, it is not known what drives this weight loss.

2. Interpretation: The findings indicate that cognitive ability and BMI stabilize each other in normative cognitive aging. However, this stabilizing effect is missing in preclinical dementia, where cognitive ability does not predict change in BMI. This finding indicates that weight loss in preclinical dementia is driven by mechanisms other than declining cognitive abilities.

3. Future directions: Future research may investigate other potential drivers of weight loss during the preclinical dementia phase to better understand the obesity paradox and the physiological effects of the dementia process.

\section{HIGHLIGHTS}

- We examined reciprocal relations between body mass index and cognition over time

- Analyses were stratified by dementia status within 5 years of last follow-up

- During normal cognitive aging, body mass index and cognition stabilize each other

- In those who developed dementia, cognition did not predict change in body mass index

- Thus, declining cognition does not drive weight loss in preclinical dementia

Design), episodic memory (Thurstone's Picture Memory Task), and processing speed (Symbol Digit). ${ }^{12}$ The reliability of the four tests range between 0.82 and $0.95 .^{12}$ More details about the cognitive tests are provided in Supporting information Appendix A. Principal component analysis, based on the individual tests, was done to create a measure of general cognitive ability, which was standardized relative to means and variances in the first testing occasion. ${ }^{13}$ All cognitive measures were transformed into T-scores (mean 50 and SD of 10) prior to analyses, scaled to the first in-person testing occasion. General cognitive ability, spatial ability, episodic memory, and processing speed were included in this study. Verbal ability could not be included due to issues with model convergence. Individual trajectories were comparable across studies for all cognitive measures (Figure S1). 


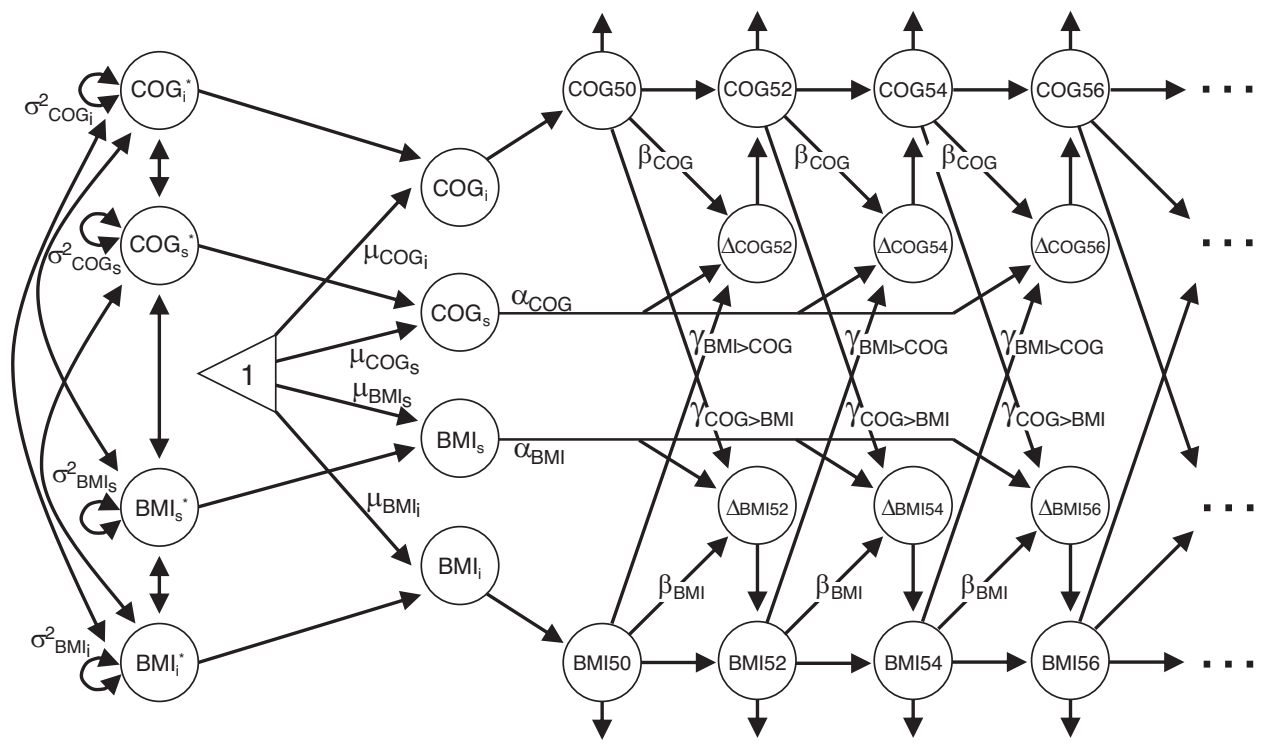

Univariate change in cognitive abilities

$\Delta_{\mathrm{COGt}}=\alpha_{\mathrm{COG}}{ }^{*} \mathrm{COG}_{\mathrm{S}}+\beta_{\mathrm{COG}}{ }^{*}\left(\mathrm{COG}_{\mathrm{t}-1}\right)$

Univariate change in BMI:

$\Delta_{\mathrm{BMIt}}=\alpha_{\mathrm{BMI}}{ }^{*} \mathrm{BMI}_{\mathrm{S}}+\beta_{\mathrm{BMI}}{ }^{*}\left(\mathrm{COG}_{\mathrm{t}-1}\right)$
Bivariate change in cognitive abilities:

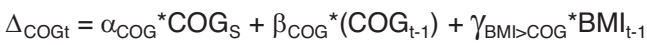

Bivariate change in $\mathrm{BMI}$ :

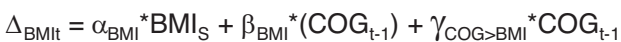

FIGURE 1 Path diagram of the bivariate dual change score model of body mass index (BMI) and cognition. Level of BMI and cognitive abilities (COG) are modeled in each age category (BMI50, BMI52...; COG50, COG52...). BMI, $\mathrm{BMI}_{\mathrm{S}}, \mathrm{COG}_{\mathrm{i}}$, and $\mathrm{COG}_{\mathrm{S}}$ represent intercept level and slope of $\mathrm{BMI}$ and cognitive ability; $\mu_{\mathrm{BMI} 50}, \mu_{\mathrm{BMIS}}, \mu_{\mathrm{COG} 50}$, and $\mu_{\mathrm{COGS}}$ their estimated mean levels; and $\sigma^{2} \mathrm{BMII}, \sigma^{2} \mathrm{BMIs}, \sigma^{2}$ coGi, and $\sigma^{2}$ coGs their variances. $\alpha_{\mathrm{BMI}}$ and $\alpha_{\mathrm{COG}}$ represent the constant change and is fixed at 1 , whereas $\beta_{\mathrm{BMI}}$ and $\beta_{\mathrm{COG}}$ represent the proportional change from one time point to the next. The coupling effect of BMI on cognition is represented by the $\gamma_{\mathrm{BMI}}>\mathrm{COG}$ parameter, and that of cognition on BMI by the $\gamma_{\mathrm{COG}}>\mathrm{BMI}$ parameter. The equations on the left show the univariate change in cognition and BMI, respectively. Univariate change in $\mathrm{BMI}$ at age $={ }_{t}$, is here determined by the constant change $\left(\alpha_{\mathrm{BMI}}{ }^{*} \mathrm{BMI}_{\mathrm{S}}\right)$ plus the proportional change multiplied by BMI level at the preceding time point $\left(\beta_{\mathrm{BMI}}{ }^{*} \mathrm{BMI} \mathrm{I}_{\mathrm{t}-1}\right)$. When a breakpoint is included in the model, the $\beta_{\mathrm{BMI}}$ parameter can differ before and after the breakpoint. The equations on the right show bivariate change in cognition and BMI, respectively. For change in BMI, the effect of cognitive ability is considered by adding the coupling effect, which is multiplied by cognitive ability at the preceding time point, to the formula $\left(\gamma_{\mathrm{COG}>\mathrm{BMI}}{ }^{*} \mathrm{COG}_{\mathrm{t}-1}\right)$. As with the $\beta_{\mathrm{BMI}}$ parameter, the $\gamma_{\mathrm{COG}>\mathrm{BMI}}$ parameter can differ before and after a breakpoint. Univariate and bivariate changes in cognitive abilities are determined by the corresponding formulas and parameters. Figure 1 is adapted from https://doi.org/10.1038/s41598-021-86667-4

\subsection{Dementia status}

All studies entailed a dementia evaluation, and, in addition, dementia after the end of study was retrieved from nationwide healthcare registers (the National Patient Register, Cause of Death Register, and Prescribed Drug Register), as described in detail previously. ${ }^{11}$ Individuals diagnosed with dementia either during or within 5 years of last study participation were categorized into the dementia group; those not diagnosed during that period were categorized as cognitively intact. For analyses, we used only cognitive measures prior to dementia diagnosis.

\subsection{Covariates}

Information about age at each testing occasion, sex, and education was available. Education was categorized into "7 years or less" or "more than 7 years," corresponding to basic or more than basic education at the time.

\subsection{Statistical analyses}

Dual change score models (DCSMs) ${ }^{14,15}$ were conducted in Mplus ${ }^{16}$ to study whether level of BMI predicts change in cognitive abilities, and whether level of cognitive abilities predicts change in BMI.

The data were first split into 2-year intervals according to age when $\mathrm{BMI}$ and cognitive ability were measured. The youngest participants were 50-years-old at baseline, and due to sparsity of data from age 90 onward we included measurements from age 50 through 89, categorized into intervals from age 50 to 51 through 88 to 89 . Sex and education were adjusted for by regressing them on intercept levels and slopes, and age (in 2-year bins) used as the underlying timescale. Relatedness between twins was accounted for with robust standard errors. All model comparisons described below were done by applying the loglikelihood difference test with a maximum likelihood robust (MLR) correction for scaling factors. ${ }^{17}$ Statistical significance threshold was set at $\alpha=0.05$.

A path diagram describing the DCSM model along with formulas for calculating change is provided in Figure 1. Univariate DCSMs of linear 
and non-linear change in BMI and cognitive abilities were first applied. In addition to estimating the mean intercept level $\left(\mu_{\mathrm{BMI}}, \mu_{\mathrm{COGi}}\right)$ and linear slope ( $\left.\mu_{\mathrm{BMIs}}, \mu_{\mathrm{COG}}\right)$, the model estimates change from one time point to the next $\left(\Delta_{\mathrm{BMIt}}\right.$ and $\left.\Delta_{\mathrm{COG}}\right)$ as a function of a static linear slope $\left(\mathrm{BMI}_{\mathrm{S}}, \mathrm{COG}_{\mathrm{S}}\right)$ plus a proportional change $\left(\beta_{\mathrm{BMI}}, \beta_{\mathrm{COG}}\right)$, which is relative to the previous level and thus a measure of non-linear change from one time point to the next.

The linear slope and proportional change are assumed to be constant over time. To test for differences in rate of change, we included breakpoints in the univariate models after age 65, 69, and 75, which allows for different proportional change before and after the breakpoint. The best fitting breakpoint model was selected based on Akaike information criteria (AIC), and compared to a null model without a breakpoint.

Next, bivariate DCSMs were applied, modeling the dynamic association between BMI and cognitive abilities. In addition to the parameters in the univariate DCSM, the bivariate model also estimates crosstrait coupling parameters. These coupling parameters are estimates of how change in cognition from one time point to the next is influenced by BMI level at the previous time point $\left(\gamma_{\mathrm{BMI}}>\mathrm{COG}\right)$, and vice versa $\left(\gamma_{\mathrm{COG}}>\mathrm{BMI}\right)$. As with the univariate proportional parameters, these coupling parameters are assumed to be constant across time but can differ before and after a breakpoint.

By comparing models with and without the coupling parameters, the temporal order of changes can be tested. First, a full-coupling (bidirectional) model, with both coupling parameters included, was applied. This was compared to a no-coupling model with neither coupling parameter included to test for any association, and to two models with only one of the coupling parameters to test for unidirectional effects. These model comparisons were carried out both in the full sample and by dementia status.

Finally, we tested for differences in specific model parameters by dementia status. We first examined group differences in univariate parameters, by comparing a model where all univariate parameters were allowed to vary freely in the two groups to models where (1) residual variances, (2) variances and covariances, (3) proportional change parameter, (4) mean intercept, and (5) mean slope were constrained in a stepwise manner. We then examined group differences in bivariate parameters in a similar manner, by comparing a model where all univariate and bivariate parameters were free to vary freely across groups to models where (1) coupling parameters, (2) residual covariance, and (3) cross-trait covariance between intercepts and slopes (ie, all bivariate parameters) were constrained in a stepwise manner.

\section{RESULTS}

\subsection{Study population}

Among the 2057 study participants, 1959 individuals had BMI and/or cognition information collected between age 50 and 89 and were included in the analysis. Two individuals were excluded due to missing information about education, leaving a final analysis sample of 1957 individuals. Stratifying on dementia status yielded 1498 individuals in the cognitively intact group and 459 individuals in the group diagnosed with dementia within 5 years of last cognitive measurement. Sample characteristics are presented in Table 1.

\subsection{Univariate trajectories of BMI and cognitive abilities}

Models allowing for nonlinear age trajectories had a significantly better fit for all outcomes $(P \leq 0.01)$. For BMI, a model with a breakpoint at age 69 had the best fit, and for all cognitive domains, a breakpoint at age 65 had the best fit (based on the AIC). These breakpoints were selected for all further analyses of respective trait.

\subsubsection{Full sample}

The full univariate model estimates are presented in Table S1. The predicted mean BMI level at age 50 was 25.19 in the full sample, with a negative overall linear slope $\left(\mu_{\mathrm{BMIS}}=-1.337\right)$. Significant buffering effects $\left(\beta_{\mathrm{BMI} \leq 69}=0.058, \beta_{\mathrm{BMI} \geq 69}=0.045\right)$ indicate that higher $\mathrm{BMI}$ predicted steeper increase from age 50 to 69 , and less decline from age 69 to 89 (Table S1, univariate; Figure 2 left panel, dashed lines).

TAB LE 1 Descriptive statistics of the full sample and stratified by dementia status

\begin{tabular}{llll} 
& Full sample & Cognitively intact & Dementia \\
\hline Individuals, $N$ & 1957 & 1498 & 459 \\
\hline Age at baseline, mean (range; SD) & $72.5(50.1-89.9 ; 10.2)$ & $71.4(50.1-89.9 ; 10.5)$ & $76.0(51.6-89.8 ; 8.3)$ \\
\hline Follow-up time, mean (range; SD) & $8.0(0-27.0 ; 7.3)$ & $8.3(0-27.0 ; 7.5)$ & $7.1(0-26.2 ; 6.8)$ \\
\hline Testing occasions, mean (range; SD) & $3.4(1-10.0 ; 2.2)$ & $3.5(1-10.0 ; 2.2)$ & $3.1(0-9.0 ; 1.9)$ \\
Women, $N(\%)$ & $1155(59.0)$ & $868(57.9)$ & $287(62.5)$ \\
\hline Lower education, $N(\%)$ & $1107(56.6)$ & $814(54.3)$ & $293(63.8)$ \\
\hline
\end{tabular}

Note. N, number; SD, standard deviation.

Descriptive statistics for the full sample, and separately for individuals who remain cognitively intact and those who are diagnosed with dementia during or within 5 years after the study. The number (\%) of individuals is presented for categorical variables and the mean level (range and SD) for continuous variables. 


\section{(A) BMI and general cognitive ability}

- Cognitively intact - Dementia

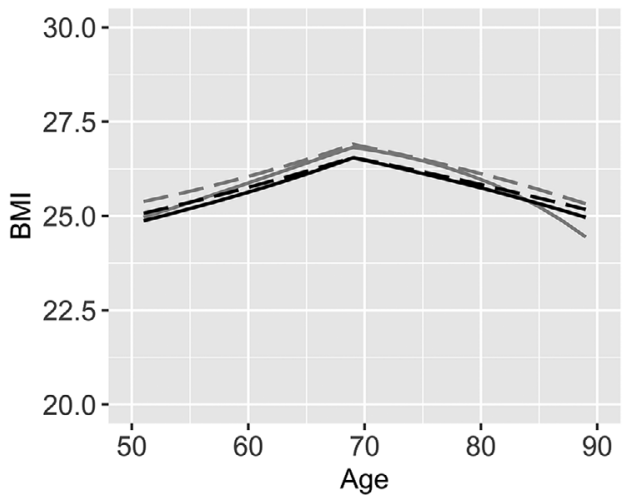

(B) BMI and spatial ability

- Cognitively intact - Dementia

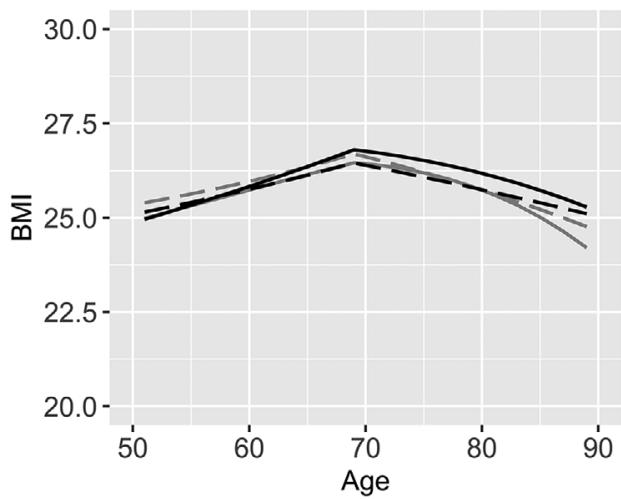

(C) BMl and processing speed

- Cognitively intact - Dementia

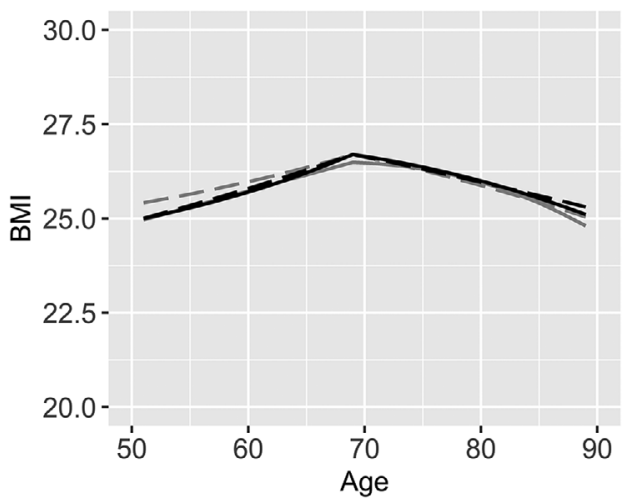

- Cognitively intact - Dementia

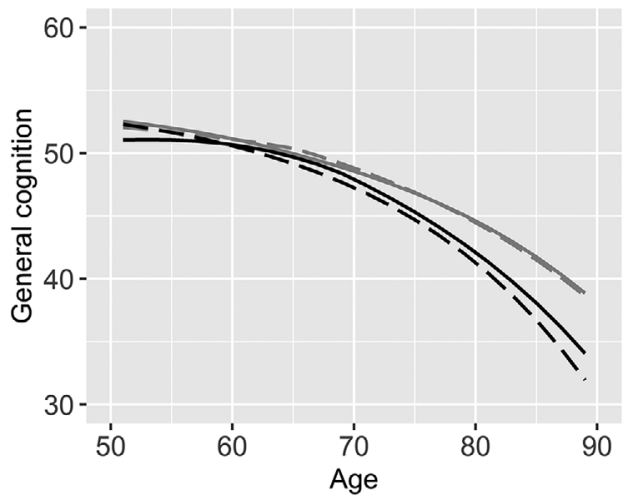

- Cognitively intact - Dementia

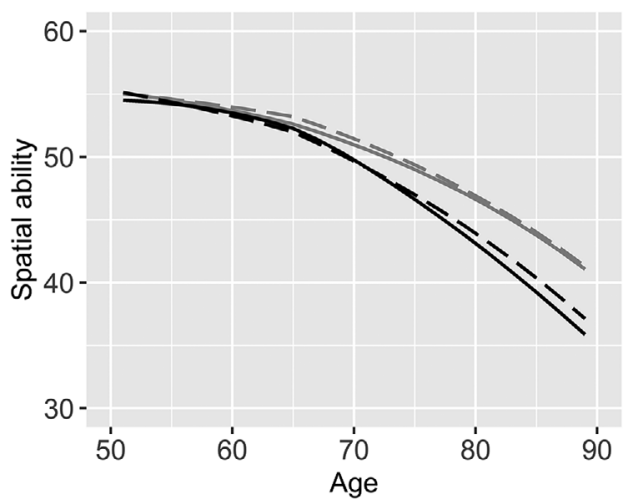

- Cognitively intact - Dementia

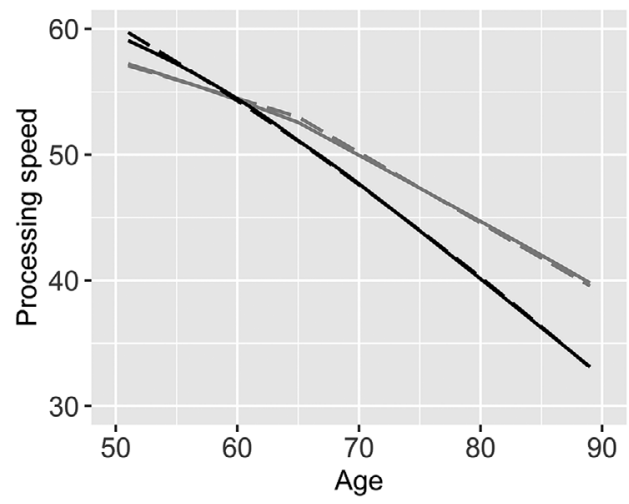

FIGURE 2 Longitudinal trajectories of change in body mass index (BMI) and cognitive abilities from age 50 to 89 , with and without the bivariate coupling parameter. Trajectories from the no-coupling dual change score model are shown in dashed lines, and those from the full coupling dual change score model in solid lines. Trajectories of individuals who remain cognitively intact are shown in gray, and those who were diagnosed with dementia within 5 years after last cognitive measure in black. Models were adjusted for sex and education, and a breakpoint was included to allow for differences in the proportional and coupling effects before and after age 69 in the trajectories of BMI and after age 65 in the trajectories of cognitive ability 
Mean general cognitive ability at age 50 was 51.901 (Table S1a, univariate). There was a negative linear slope $\left(\mu_{\mathrm{BMIS}}=-5.739\right)$, but with buffering from proportional effects $\left(\beta_{\mathrm{COG}<65}=0.107\right.$, $\left.\beta_{\mathrm{COG}>65}=0.103\right)$ resulting in a slight decline from age 50 to 65 , followed by a steeper decline from age 65 to 89 (Figure 2A right panel, dashed lines). Because proportional effects are multiplied with the cognitive level at the preceding occasion, it means that higher cognitive ability predicted less decline. It is also important to note that this means that declining cognitive ability can be accelerated due to increasingly weaker buffering effects with lower cognitive levels.

Pattern of change in spatial ability was similar to that in general cognitive ability (Figure 2B right panel, dashed lines; Table S1b, univariate). Change in episodic memory was driven by a strongly negative linear slope, but with substantial buffering from the proportional effects leading to stable levels from age 50 through 65 , followed by a gradual decline after 65 (Table S1c, univariate). Processing speed had little buffering from proportional effects and declined already between age 50 and 64, after which decline was more substantial (Figure 2D right panel, dashed lines; Table S1d, univariate).

\subsection{2 | By dementia status}

Compared to those who remained cognitively intact, individuals who were later diagnosed with dementia had significantly lower predicted BMI (estimate difference 0.244 at age 50; Table 2, univariate), but did not differ in terms of longitudinal rate of change in BMI (mean slope or proportional effects, Table 2, univariate).

For processing speed, the linear decline was significantly steeper among those who developed dementia than those who remained cognitively intact (Table $2 \mathrm{C}$ right panel, dashed lines). No statistically significant group difference in rate of change was present for the other cognitive domains, although plots generally suggest a steeper decline in the dementia group (Figure 2A,B right panel, dashed lines). Thus compared to the cognitively intact group, individuals wo were subsequently diagnosed with dementia had lower cognitive ability at later ages, although their estimated scores at age $\mathbf{5 0}$ were significantly higher for general cognitive ability and processing speed but lower for spatial ability and episodic (Table 2 and Table S1c, univariate estimates).

\subsection{Bivariate trajectories of $\mathrm{BMI}$ and cognitive abilities}

\subsubsection{Full sample}

Comparisons of models with and without the coupling parameters are presented in Table 3. The relationships between BMI and general cog nitive ability and BMI and processing speed were of a bidirectional nature, with cognitive ability driving change in BMI and BMI driving change in cognitive ability (Table 3 ). The relationship between BMI and spatial ability was of a unidirectional nature in the full sample, where spatial ability drives change in BMI, but BMI does not drive change in spatial ability. There was no statistically significant effect of coupling between BMI and episodic memory (Table 3), and no further tests were thus carried out to study the association. The model estimates for the full sample are presented in Table S1.

\subsection{2 $\quad$ By dementia status}

Longitudinal trajectories of change in BMI and cognitive abilities, with and without considering the coupling parameters, are shown in Figure 2. The no-coupling trajectories correspond to the univariate trajectories, and the full-coupling trajectories show change in BMI when considering the effect of cognitive ability, and vice versa.

Trajectory estimates from bivariate models are presented in Table 2, with full model estimates presented in Table S1. Likelihood ratio tests (LRTs) demonstrated significant group differences for the effect of general cognitive ability on change in BMI, and for the effect of BMI on general cognitive ability and spatial ability (Table 2 ).

\section{Cognitively intact}

Among individuals who remained cognitively intact, the nature of the relationships between $\mathrm{BMI}$ and cognitive abilities were the same as in the full sample, namely that the associations between $\mathrm{BMI}$ and general cognitive ability and $\mathrm{BMI}$ and processing speed were of a bidirectional nature, whereas spatial ability drives change in BMI but not the opposite (Table 3).

By comparing trajectory estimates from univariate and bivariate models, the effect of the coupling mechanisms can be studied. The coupling parameters should then be interpreted together with changes in the linear slope and proportional effects. When the effect of general cognitive ability, as well as that of spatial ability and processing speed, was considered, BMI at age 50 was lower, the negative linear slope steeper, and buffering from proportional effects after age 69 weaker (Table 2). However, there was an additional buffering effect from coupling parameters, both before and after age 69. Because the coupling parameters are multiplied with level of the other variable at the preceding occasion, this means that higher cognitive ability predicts slight increases in BMI from age 50 to 69, and less decline in BMI after age 69. In Figure 2 (left panel), this is seen as steeper increase from age 50 to 69 , followed by more stable level after age 69 . However, Figure 2 shows a steeper decline at later ages when the effect of cognition is considered, likely due to lower levels of general cognitive ability limiting buffering from coupling effect.

When the effect of BMI was considered, general cognitive ability and processing speed were slightly higher at age 50 , but the linear slope showed steeper decline (Table 2A,C). However, this was buffered by stronger proportional effects, especially before age 65 . The coupling effects from BMI added to the decline before age 65 , but buffered against it after age 65. In Figure 2 (right panel), this is seen as a slightly steeper decline in cognitive abilities from age 50 to 65 , and slightly less decline from age 65 to 89 when the effect of $\mathrm{BMI}$ is considered.

The coupling effects of BMI on general cognitive ability and processing speed were negative from age 50 to 65 , meaning that higher $\mathrm{BMI}$ 


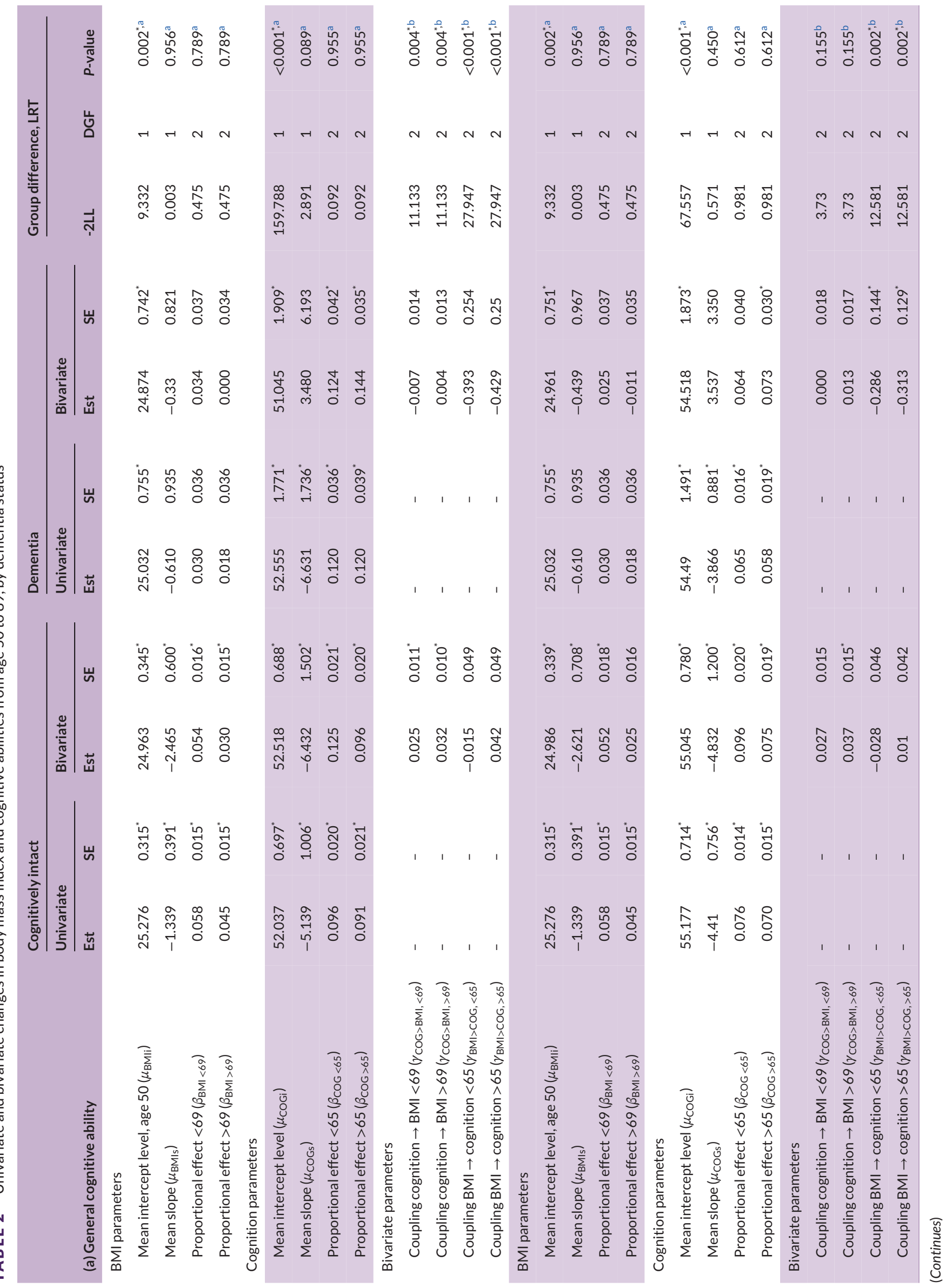



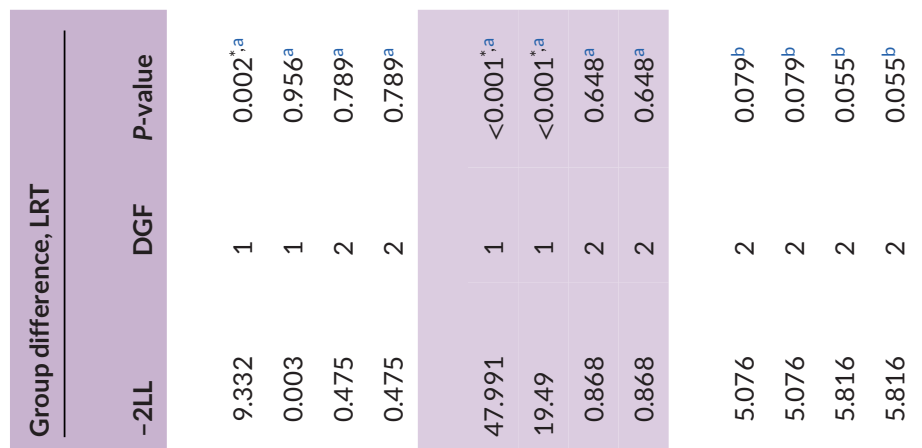

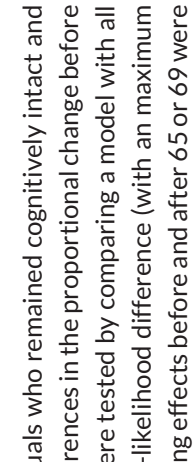

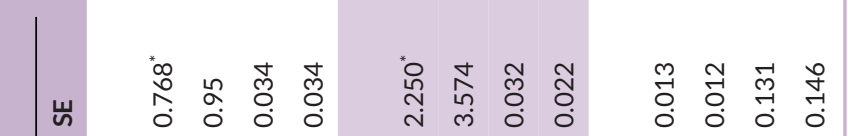

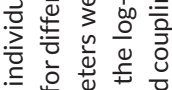

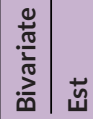

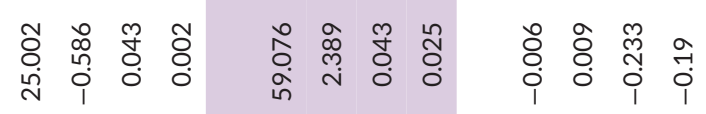

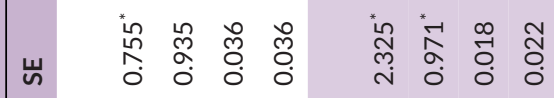

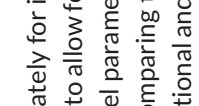

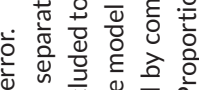

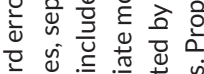

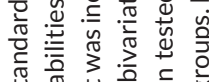

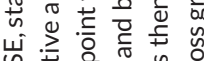

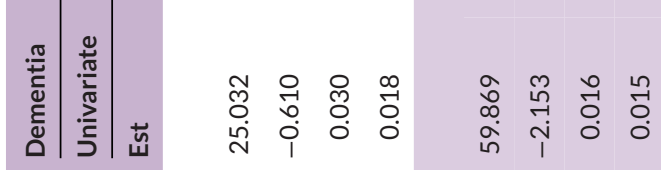

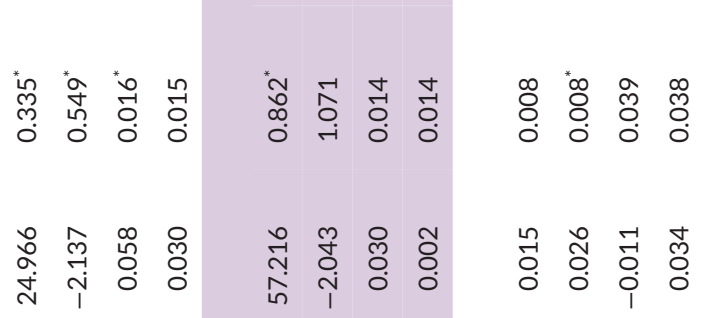

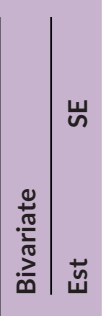

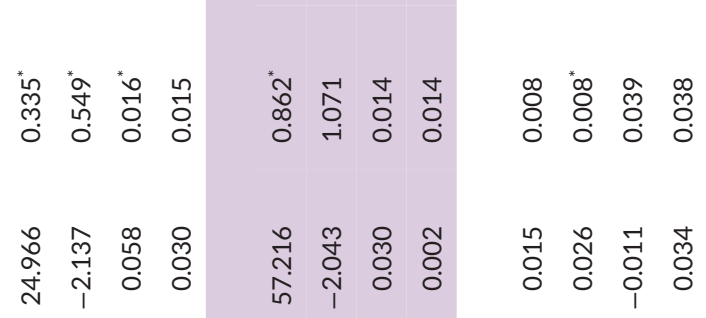

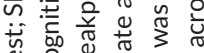

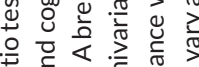

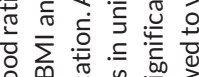

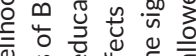

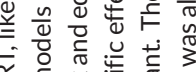

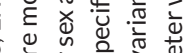

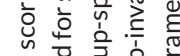

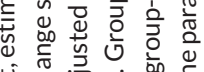

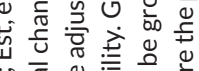

है

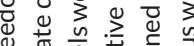

辛.

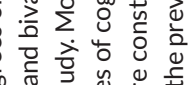

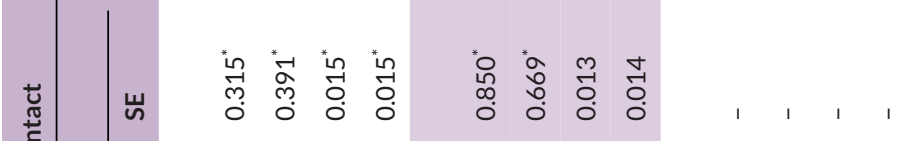

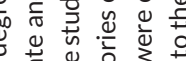

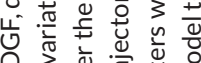

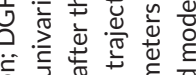

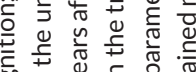

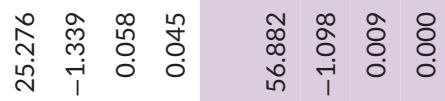

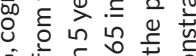

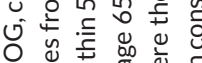

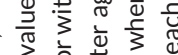

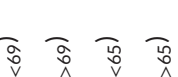

然

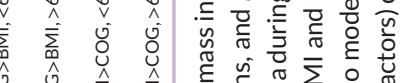

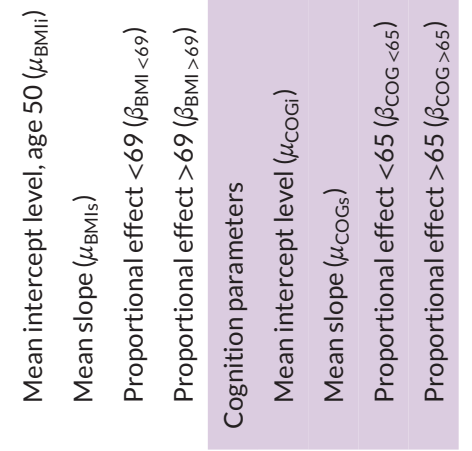

全

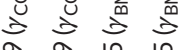

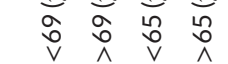

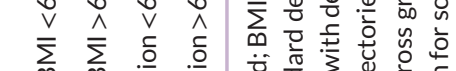

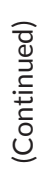

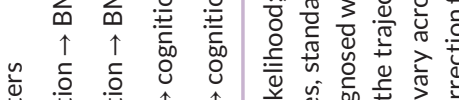

过

虫

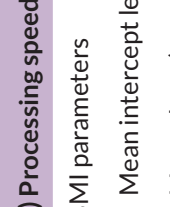

党

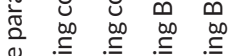

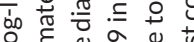

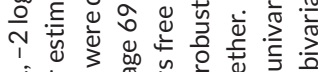

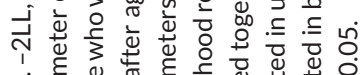

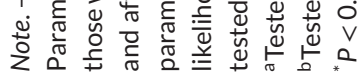


TABLE 3 Bivariate model comparisons

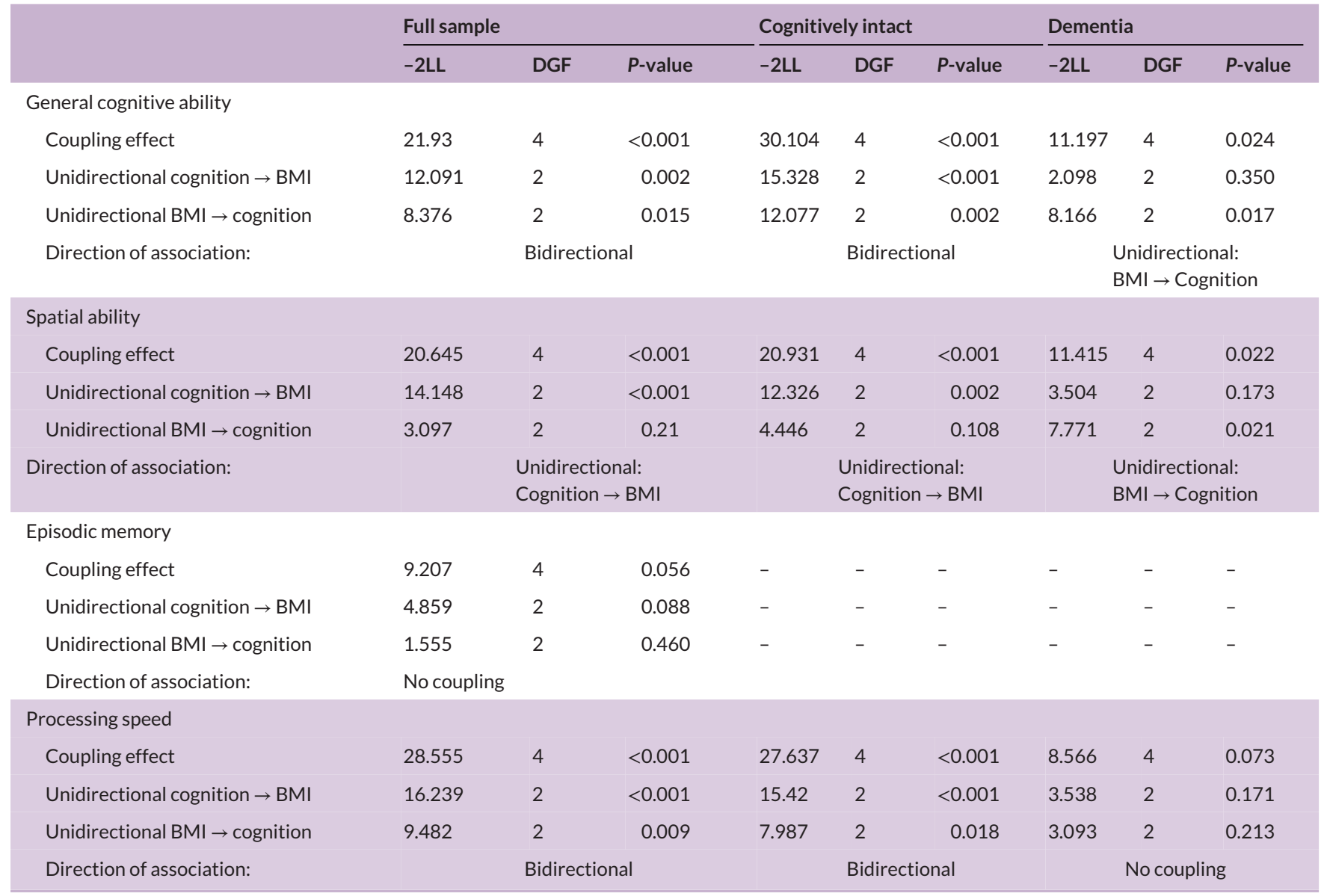

Note. -2LL, -2 log likelihood; DGF, degrees of freedom.

Comparisons of bivariate dual change score models of BMI and cognitive abilities. To test for evidence of a coupling effect, a full-coupling (bidirectional) model was compared to a no-coupling model. Second, unidirectional effects were examined by comparing unidirectional models to the full-coupling model. The significance of the increase in model fit was computed by comparing the log-likelihood difference (with an MLR correction for scaling factors) of the models. Associations were first examined in the full sample, and then, when an association was present, separately in the cognitively intact and dementia group.

predicts a steeper decline in cognitive ability, but positive from age 65 through 89 , with higher BMI predicting less decline in cognitive ability (Table 2A,C). Taken together, this indicates that in older ages a stable $\mathrm{BMI}$ predicts a stable cognitive ability and vice versa.

\section{Dementia}

Among individuals who were later diagnosed with dementia the associations between BMI and general cognitive ability and BMI and spatial ability were unidirectional, with BMI driving change in cognitive ability, but not the opposite (Table 3). Both before and after age 69, higher BMI predicted steeper decline in cognition. This result is indicated by a positive overall linear slope in the bivariate model (in contrast to the univariate model, where the linear slope was negative; Table $2 \mathrm{~A}, \mathrm{~B}$ ), counteracted by strongly negative coupling effects (multiplied by BMI level at the preceding occasion), which thus drives the decline. The buffering from proportional effects was less affected by the influence of BMI. There was no statistically significant coupling present for the association between $\mathrm{BMI}$ and processing speed (Table 3).
As visualized in Figure 2, considering the effect of BMI led to a more stable cognitive ability from age 50 to 65 , followed by a comparable decline in general cognition but a steeper decline in spatial ability.

\section{DISCUSSION}

We here used the powerful DCSM to study the dynamic relationship between $\mathrm{BMI}$ and cognitive abilities from age 50 through 89 , and demonstrated that the nature of the association differed between individuals who remained cognitively intact and those who were later diagnosed with dementia. Among individuals who were subsequently diagnosed with dementia, higher BMI predicted steeper decline in general cognitive ability, and in spatial ability in particular, throughout midlife and late life, whereas level of cognitive ability did not predict change in BMI. Among those who remained cognitively intact, for general cognitive ability, and processing speed in particular, the associations with $\mathrm{BMI}$ were of a bidirectional nature, such that higher BMI predicted a steeper decline in cognitive abilities before age 65 but-in contrast to 
the preclinical dementia group-buffered against decline after age 65 In turn, general cognitive ability, and particularly level of processing speed and spatial ability, drives change in BMI, with higher cognitive ability predicting more increase in midlife and less decline in late life. No coupling effect between BMI and episodic memory was identified.

The differences in the results by dementia status highlight that the relationship between $\mathrm{BMI}$ and cognitive abilities is dysfunctional in preclinical dementia. Among individuals who remain cognitively intact, stable cognitive abilities predict stability in BMI, and a stable BMI predicts stability in cognitive abilities in older ages. Among individuals who developed dementia, on the other hand, these stabilizing effects are missing. Instead, we see a decline in cognitive ability, which is largely a function of the coupling effects from BMI, with too little buffering from the cognitive level itself to compensate for the negative effects. This indicates that lower cognitive ability, per se, does not explain weight loss in preclinical dementia. Rabin and colleagues ${ }^{18}$ demonstrated that a higher amyloid beta burden predicted more decline in BMI in a sample of cognitively normal individuals at baseline. It is notable that the results persisted in models adjusted for cognitive performance. Müller et al. have shown that $\mathrm{BMI}$ also declines 10 to 20 years prior to the expected onset of autosomal dominant Alzheimer's disease (AD). ${ }^{19}$ The decline began well before any clinical or cognitive symptoms were present, and is thus not an effect of cognitive impairment, but the authors did note that BMI was associated with lower cognitive performance, still within normal ranges. This also shows that decline in BMI is not only an effect of older age, but specifically linked to the preclinical AD process. Taken together, these findings and ours indicate that decline in $\mathrm{BMI}$ seen in the preclinical dementia phase is likely not driven by declining cognitive ability. This highlights that weight loss in late life may be a warning sign of dementia, even in the absence of declining cognitive abilities. The biological mechanisms driving the decline in $\mathrm{BMI}$ remains unclear, but are postulated to be a result of preclinical AD pathology affecting hypothalamic regulation of body weight and systemic metabolism, or that it affects the olfactory system or mental health. ${ }^{18,19}$

This study was built on a well-characterized sample with objectively measured BMI, robust cognitive measures, and dementia assessed during the study as well as through register linkage. The sample allowed us to study change from age 50 through 89 and to apply the powerful DCSM. The study is not without limitations. The sample size, although phenotypically rich, was limited. We could therefore not look closer at, for example, sex-specific or weight-category specific effects, or investigate the effects of other medical risk factors such as cardiometabolic traits. BMI is an imperfect measure of overweight, and does not consider body fat distribution. However, it is a robust and readily available measure, and is expected to capture late-life weight changes well. As in all studies of older adults, poor health may lead to survival bias and attrition rate. ${ }^{20}$ This issue may be more important among individuals later diagnosed with dementia, highlighting the importance of supplementing dementia diagnosed during the studies with register linkage after the end of follow-up. It should be noted, however, that although dementia information from healthcare registers has excellent speci- ficity, the sensitivity is rather low, ${ }^{21}$ and some dementia diagnoses may have been missed.

In conclusion, we here show that the longitudinal association between BMI and cognitive abilities differ among individuals who develop dementia compared to those who remain cognitively intact. Although BMI and cognitive abilities stabilize each other when cognition remains intact, this buffering effect is missing in the preclinical phase of dementia, where only a negative effect of higher BMI remains. This is in agreement with previous evidence indicating that weight loss in preclinical dementia is not an effect of cognitive impairment, but may rather be an effect downstream of AD pathology. ${ }^{18,19}$

\section{ACKNOWLEDGMENTS}

This work was supported by the Swedish Research Council for Health, Working Life and Welfare (2018-01201); the Swedish Research Council (2016-03081); and the National Institutes of Health (NIH AG060470). We acknowledge the Swedish Twin Registry for access to data. The Swedish Twin Registry is managed by Karolinska Institutet and receives funding through the Swedish Research Council under grant no. 2017-00641. SATSA was supported by the NIH (grants AG04563 and AG10175), the MacArthur Foundation Research Network on Successful Aging, the Swedish Research Council for Working Life and Social Research (FAS; Grants 97:0147:1B, 2009-0795), and the Swedish Research Council (825-2007-7460 and 825-2009-6141). OCTO-Twin was supported by the NIH (grant R01 AG08861). GENDER was supported by the MacArthur Foundation Research Network on Successful Aging, The Axel and Margaret Ax:son Johnson's Foundation, The Swedish Council for Social Research, and the Swedish Foundation for Health Care Sciences and Allergy Research.

\section{CONFLICTS OF INTEREST}

The authors declare no conflicts of interest.

\section{ORCID}

Ida K. Karlsson (iD https://orcid.org/0000-0003-3605-7829

\section{REFERENCES}

1. Aisen PS, Cummings J. On the path to 2025: understanding the Alzheimer's disease continuum. Alzheimers Res Ther. 2017;9:60.

2. Peters R, Peters J, Booth A, Anstey KJ. Trajectory of blood pressure, body mass index, cholesterol and incident dementia: systematic review. Br J Psychiatry. 2020;216:16-28.

3. Danat IM, Clifford A, Partridge M, et al. Impacts of overweight and obesity in older age on the risk of dementia: a systematic literature review and a meta-analysis. J Alzheimers Dis. 2019;70:S87-S99.

4. Dye L, Boyle NB, Champ C, Lawton C. The relationship between obesity and cognitive health and decline. Proc Nutr Soc. 2017;76:443-454.

5. Dahl AK, Hassing LB. Obesity and cognitive aging. Epidemiol Rev. 2013;35:22-32.

6. Dahl AK, Reynolds CA, Fall T, Magnusson PK, Pedersen NL. Multifactorial analysis of changes in body mass index across the adult life course: a study with 65 years of follow-up. Int J Obes. 2014;38:1133-1141.

7. Zagai U, Lichtenstein P, Pedersen NL, Magnusson PKE. The Swedish Twin Registry: content and management as a research infrastructure. Twin Res Hum Genet. 2019;22:672-680. 
8. Finkel D, Pedersen N. Processing speed and longitudinal trajectories of change for cognitive abilities: the Swedish adoption/twin study of aging. Neuropsychology, development, and cognition Section B, Aging, neuropsychology and cognition. 2004;11:325-345.

9. Gold CH, Malmberg B, McClearn GE, Pedersen NL, Berg S. Gender and health: a study of older unlike-sex twins. J Gerontol: Soc Sci. 2002;57:S168-S176.

10. McClearn GE, Johansson B, Berg S, et al. Substantial genetic influence on cognitive abilities in twins 80 or more years old. Science. 1997;276:1560-1563.

11. Karlsson IK, Lehto K, Gatz M, Reynolds CA, Dahl Aslan AK. Agedependent effects of body mass index across the adult life span on the risk of dementia: a cohort study with a genetic approach. BMC Medicine. 2020;18:131.

12. Pedersen NL, Plomin R, Nesselroade JR, McClearn GE. A quantitative genetic analysis of cognitive abilities during the second half of the life span. Psychol Sci. 1992;3:346-353.

13. Finkel D, Reynolds CA, McArdle JJ, Gatz M, Pedersen NL. Latent growth curve analyses of accelerating decline in cognitive abilities in late adulthood. Dev Psychol. 2003;39:535-550.

14. McArdle JJ, Hamagami F, Meredith W, Bradway KP. Modeling the dynamic hypotheses of Gf-Gc theory using longitudinal life-span data. Learning and Individual Differences. 2000;12:53-79.

15. McArdle J, Cudeck R, Toit S, Sorbom D. Structural equation modeling: present and future. In: Cudeck R, duToit S, Sorbom D, eds. A Latent Difference Score Approach to Longitudinal Dynamic Structural Analyses. 2001:342-380.

16. Muthén LKaM BO. Mplus User's Guide. Eighth Edition ed. Los Angeles, CA: Muthén \& Muthén; 1998-2017.
17. Satorra A, Bentler PM. A scaled difference chi-square test statistic for moment structure analysis. Psychometrika. 2001;66:507-514.

18. Rabin JS, Shirzadi Z, Swardfager W, et al. Amyloid-beta burden predicts prospective decline in body mass index in clinically normal adults. Neurobiol Aging. 2020;93:124-130.

19. Muller S, Preische O, Sohrabi HR, et al. Decreased body mass index in the preclinical stage of autosomal dominant Alzheimer's disease. Sci Rep. 2017;7:1225.

20. Kelfve S, Fors S, Lennartsson C. Getting better all the time? Selective attrition and compositional changes in longitudinal and life-course studies. Longitudinal and Life Course Studies. 2017;8.

21. Rizzuto D, Feldman AL, Karlsson IK, Dahl Aslan AK, Gatz M, Pedersen NL. Detection of dementia cases in two Swedish Health Registers: a validation study. J Alzheimers Dis. 2018;61:1301-1310.

\section{SUPPORTING INFORMATION}

Additional supporting information may be found online in the Supporting Information section at the end of the article.

How to cite this article: Karlsson IK, Zhan Y, Gatz M, Reynolds CA, Dahl Aslan AK. Change in cognition and body mass index in relation to preclinical dementia. Alzheimer's Dement.

2021;7:e12176. https://doi.org/10.1002/trc2.12176 